\title{
Obtención de productos de valor añadido mediante la hidrogenólisis de glicerina sin aporte externo de hidrógeno
}

\author{
Raquel Raso, Lucía García, Joaquín Ruiz, Miriam Oliva, Jesús Arauzo \\ Grupo de Procesos Termoquímicos (GPT) \\ Instituto de Investigación en Ingeniería de Aragón (I3A) \\ Universidad de Zaragoza, Mariano Esquillor s/n, 50018, Zaragoza, Spain. \\ Tel.+34-976762707, e-mail: rroka@unizar.es
}

\section{Resumen}

En este trabajo se estudió la actividad catalítica de los catalizadores de $\mathrm{Ni}-\mathrm{Al}-\mathrm{Fe}$ en la hidrogenólisis de glicerina sin adición de hidrógeno. Dichos catalizadores se prepararon por el método de coprecipitación cambiando la relación molar Al/Fe. Además, se analizó la posible lixiviaciación de los metales en la reacción.

\section{Introducción}

Actualmente, la producción de biodiésel es un tema atractivo debido a la elevada concienciación sobre los problemas medioambientales y el agotamiento de los combustibles fósiles. Por ende, se ha incrementado la producción de dicho biocombustible. El biodiésel se puede obtener mediante la transestificación de triglicéridos como es el aceite vegetal. Durante el proceso de producción del biocombustible, por 10 toneladas de biodiésel producido se genera 1 tonelada de glicerina como subproducto. Por lo tanto, se genera un excedente de producción de glicerina que afectaría en gran medida a la economía de la producción de biodiésel. Es por ello por lo que, en la última década, se ha estudiado y desarrollado nuevas alternativas para valorizar la glicerina [1,2]. En este contexto, se propone la conversión de glicerina en productos de valor añadido como por ejemplo son el 1,2-propanodiol, acetol y etilenglicol mediante el proceso de hidrogenólisis de la misma sin aporte externo de hidrógeno.

El 1,2-propanodiol es un importante producto químico, utilizado principalmente en alimentos, productos famacéuticos, detergentes líquidos, entre otros usos [3]. El etilenglicol se utiliza como anticongelante y materia prima para la fabricación de poliéster [4]. El acetol tiene sus usos en la industria textil, alimentaria y cosmética. Además, se puede utilizar para obtener compuestos como el propilenglicol, acetona, entre otros [5].

En este trabajo se estudió la actividad catalítica de los diferentes catalizadores de Ni-Al-Fe preparados por el método de coprecipitación modificando la relación molar Al/Fe. También, se analizó la posible lixiviación de los metales durante la reacción del proceso de hidrogélisis de la glicerina sin aporte de hidrógeno.

\section{Sistema experimental}

Los diferentes catalizadores de Ni-Al-Fe se sintetizaron utilizando el método de coprecipitación, según lo descrito por Raso et al. [6] manteniendo constante el contenido de $\mathrm{Ni}$ al 28\% molar y cambiando la relación molar de $\mathrm{Al} / \mathrm{Fe}$. La relación molar se cambió con valores 1/0,3/1, 1/1, 1/3, 0/1 y los catalizadores se denominaron $\mathrm{Al}_{1} \mathrm{Fe}_{0}, \mathrm{Al}_{3} \mathrm{Fe}_{1}$, $\mathrm{Al}_{1} \mathrm{Fe}_{1}, \mathrm{Al}_{1} \mathrm{Fe}_{3}$ y $\mathrm{Al}_{0} \mathrm{Fe}_{1}$, respectivamente.

Los experimentos se llevaron a cabo en una instalación de laboratorio continua a pequeña escala diseñada y desarrollada por PID (Process Integral Development Eng \& Tech, Spain), descrita en trabajos anteriores [4,6,7]. El estudio de la actividad catalítica tuvo lugar en un reactor de lecho fijo durante 3 h a $227{ }^{\circ} \mathrm{C}$ y 34 bar. Se utilizó como alimentación una disolución de glicerina en agua desionizada al $10 \%$ en peso y un caudal total de 1 $\mathrm{mL} / \mathrm{min}$. Los productos gaseosos se analizaron en línea por un micro-GC, equipado con detectores de conductividad térmica (TCD) que permite cuantificar los gases producidos y utilizando el $\mathrm{N}_{2}$ como estándar interno. Los productos líquidos fueron analizados por un GC/FID y utilizando el 1-butanol como estándar interno. $\mathrm{Y}$ a su vez, los líquidos recogidos tras los experimentos fueron analizados mediante la técnica ICP-OES para determinar la cantidad de metales lixiviados. 


\section{Resultados y discusión}

Se vio que todos catalizadores eran activos para el proceso de hidrogenólisis de la glicerina. Además, se observó que los catalizadores $\mathrm{Al}_{3} \mathrm{Fe}_{1}, \mathrm{Al}_{1} \mathrm{Fe}_{1}, \mathrm{Al}_{1} \mathrm{Fe}_{3}$ fueron los que presentaron los mejores resultados de actividad catalítica que los catalizadores $\mathrm{Al}_{1} \mathrm{Fe}_{0}$ y $\mathrm{Al}_{0} \mathrm{Fe}_{1}$, siguiendo el siguiente orden: $\mathrm{Al}_{3} \mathrm{Fe}_{1}>\mathrm{Al}_{1} \mathrm{Fe}_{1}$ $>\mathrm{Al}_{1} \mathrm{Fe}_{3}>\mathrm{Al}_{1} \mathrm{Fe}_{0}>\mathrm{Al}_{0} \mathrm{Fe}_{1}$.

Finalmente, se observó que durante la reacción se lixiviaban los metales del catalizador. Esto podría deberse al $\mathrm{CO}_{2}$ disuelto y/o los compuestos oxigenados obtenidos durante el proceso de hidrogenólisis de la glicerina.

\section{Conclusiones}

En este trabajo, se estudió la actividad catalítica de los catalizadores Ni-Al-Fe preparados mediante el método de coprecipitación. Se vio que dichos catalizadores eran activos para el proceso de hidrogenólisis de la glicerina y siendo el catalizador $\mathrm{Al}_{3} \mathrm{Fe}_{1}$, el más activo. Finalmente, se observó la lixiviación de los metales en la reacción.

\section{Agradecimientos}

Los autores quieren expresar su gratitud a AEI / FEDER, UE (proyecto CTQ2017-86893-R) por el apoyo financiero recibido. También agradecen la financiación recibida del Gobierno de Aragón (Referencia T22_20R) y cofinanciado con FEDER 2014-2020 “Construyendo Europa desde Aragón”.

\section{REFERENCIAS}

[1]. MAHLIA, T., SYAZMI, Z., MOFIJUR, M., ABAS, A., BILAD, M., ONG, H. and Silitonga, A. Patent landscape review on biodiesel production: Technology updates. Renewable \& Sustainable Energy Reviews. 2020, $118 . \quad$ Available from: doi:10.1016/j.rser.2019.109526.

[2]. ZHOU, C., ZHAO, H., TONG, D., WU, L. and YU, W. Recent Advances in Catalytic Conversion of Glycerol. Catalysis Reviews-Science and Engineering. 2013, 55, 369-453. Available from: doi:10.1080/01614940.2013.816610.

[3]. DASARI, M., KIATSIMKUL, P., SUTTERLIN, W. and SUPPES, G. Low-pressure hydrogenolysis of glycerol to propylene glycol. Applied Catalysis aGeneral. 2005, 281, 225-231. Available from: doi:10.1016/j.apcata.2004.11.033.

[4]. GARCIA, L., VALIENTE, A., OLIVA, M., RUIZ, J. and ARAUZO, J. Influence of operating variables on the aqueous-phase reforming of glycerol over a $\mathrm{Ni} / \mathrm{Al}$ coprecipitated catalyst. International Journal of Hydrogen Energy. 2018, 43, 20392-20407. Available from: doi:10.1016/j.ijhydene.2018.09.119.

[5]. MOHAMAD, M.H., AWANG, R. and YUNUS, W.M.Z.W. A Review of Acetol: Application and Production. American Journal of Applied Sciences. 2011, 11, 1135-1139. Available from: doi:https://doi.org/10.3844/ajassp.2011.1135.1139.

[6]. RASO, R., GARCÍA, L., RUIZ, J., OLIVA, M. and ARAUZO, J. Aqueous phase hydrogenolysis of glycerol over $\mathrm{Ni} / \mathrm{Al}-\mathrm{Fe}$ catalysts without external hydrogen addition. Applied Catalysis B: Environmental. 2021, 283, 119598. Available from: doi:https://doi.org/10.1016/j.apcatb.2020.119598.

[7]. REMON, J., GIMENEZ, J., VALIENTE, A., GARCIA, L. and ARAUZO, J. Production of gaseous and liquid chemicals by aqueous phase reforming of crude glycerol: Influence of operating conditions on the process. Energy Conversion and Management. 2016, 110, 90-112. Available from: doi:10.1016/j.enconman.2015.11.070. 